Article

\title{
Pathotypes of Xanthomonas axonopodis pv. dieffenbachiae Isolated from Anthurium andraeanum in China
}

\author{
Shuang-Chun Li ${ }^{1,+}$, Wei-Da Zeng ${ }^{2,+}$, Xing-Wei Li ${ }^{1}$, Xiao-Yun Zhou ${ }^{2, *}$ and Qiong-Guang Liu ${ }^{1, *}$ \\ 1 State Key Laboratory of Conservation and Utilization of Subtropical Agro-Bioresources, Guangdong \\ Province Key Laboratory of Microbial Signals and Disease Control, College of Agriculture, \\ South China Agricultural University, Guangzhou 510642, China; cherrylee92@foxmail.com (S.-C.L.); \\ xingwli@163.com (X.-W.L.) \\ 2 Guangzhou Flower Research Centre, Guangzhou 510360, China; weidaz@126.com \\ * $\quad$ Correspondence: qgliu@scau.edu.cn (Q.-G.L.); xyzh28@126.com (X.-Y.Z.); Tel.: +86-20-85281803 (Q.-G.L.) \\ + These authors contributed equally to this study.
}

Received: 9 September 2018; Accepted: 29 October 2018; Published: 6 November 2018

\begin{abstract}
Anthurium blight, caused by Xanthomonas axonopodis pv. dieffenbachiae (Xad), is one of the most serious diseases of Anthurium andraeanum. However, little is known about variations in virulence between $\mathrm{Xad}$ pathotypes. Here, we examined the virulence of $68 \mathrm{Xad}$ strains isolated from 30 anthurium plants from five regions of China against five different anthurium cultivars. Seven bacterial pathotypes were identified based on disease index and incidence analyses following foliar spray or leaf-clip inoculation. The resulting disease susceptibility patterns for pathotypes I-VII were RRRSS, RRSRS, RSRSR, RRSSS, RSSRS, RSSSS, and SSSSS, respectively. Overall, $72 \%$ of tested strains belonged to pathotypes VI or VII and were highly virulent. A further $22.1 \%$ of strains showed medium-level virulence and were classed as pathotype III, IV, or V, while the remaining $5.9 \%$ of strains were pathotype I or II, showing low virulence. Further analysis revealed differences in the virulence of Xad strains from the same anthurium cultivar, with variation also observed in pathovars associated with the same cultivar from different areas. Our results reveal the diversity and complexity of the Xad population structure in China and suggest that investigation of Xad pathotypes provides useful information to guide the identification and use of resistant varieties of $A$. andraeanum.
\end{abstract}

Keywords: Anthurium andraeanum; bacterial blight; Xanthomonas axonopodis pv. dieffenbachiae; pathotype; China

\section{Introduction}

Anthurium andraeanum is a tropical, perennial, evergreen plant that is highly sought after because of its colorful flowers and unique leaf shape. A. andraeanum is widely planted in many countries, including China, with commercial-scale growing operations found in Guangdong, Zhejiang, Yunnan, Fujian, and Hainan provinces. However, the production and development of A. andraeanum are seriously impacted by a bacterial blight disease caused by Xanthomonas axonopodis pv. dieffenbachiae (Xad). Anthurium blight was first reported in Brazil in 1960 and subsequently in Hawaii in 1971 [1]. The disease was responsible for the decline of the Hawaiian anthurium industry in the 1980s [2] and the Caribbean anthurium industry in the 1990s [3]. Due to its devastating effects, Xad is considered a quarantine pest by the European and Mediterranean Plant Protection Organization, the Caribbean Plant Protection Commission, and by several independent countries [4].

Anthurium blight is easily transmitted through excess water run-off, infected soil, and direct contact with infected plant materials and tools $[5,6]$. Many studies have focused on the identification, 
classification, molecular detection, and physiological and biochemical characterization of Xad, as well as on potential disease control measures [7-10]. Physical measures to control the disease have been suggested [11] but they are expensive and require capital intensive infrastructure and good management practices, such as daily removal of infected leaves, cut flowers, and plants [12]. Several chemicals have been screened for their potential to control the disease [13], however, widespread application of pesticides leads to environmental pollution and drug resistance, amongst other issues.

It is generally agreed that the most cost-effective approach to controlling anthurium blight is the use of resistant cultivars [12]. A previous study suggested that Julia and Gemini were the most resistant cultivars, while Hearts Desire was the most susceptible to Xad infection among 15 tested anthurium cultivars [14]. In another study, eight anthurium cultivars, including Alii, ARCS, Kalapana, Marian Seefurth, Nitta, Pink Elf, Tropic Mist, and UH1060, were inoculated with Xad strain V108LRUH1. The results indicated that in susceptible cultivars, pathogenic bacteria spread rapidly into the front of the petiole, while bacteria affecting the resistant cultivars spread more slowly and rarely migrated into the petiole [15]. Screening of 10 anthurium cultivars naturally infected with bacteria in the field revealed that cultivars White Queen, Florida Red Ruffles, Florida Sweetheart, Candidum Jr., and Mrs. Arno Nehrling were resistant to bacterial blight in both greenhouse and field-based evaluations, making them good candidates for use in future breeding efforts to produce resistant cultivars [16]. The inheritance of foliar resistance to blight is quantitative, with a major role for additive genetic effects [17]. Differences in the virulence of Xad strains are directly linked to the resistance of different anthurium plants and little research has been conducted on Xad pathotypes [18]. We previously classified 66 Xad strains from Guangdong Province, China, into 14 genetic groups using repetitive element palindromic-polymerase chain reaction (Rep-PCR) analysis and found that Xad strains in China show abundant genetic variation. However, differences in virulence among the strains are less clear. In the present study, we determined the pathotypes of $68 \mathrm{Xad}$ strains isolated from anthurium plants from various regions of China, identifying seven different pathotypes. Among these, pathotypes VI and VII were the most virulent and were also the predominant pathotypes of Xad strains in China. The results of the current study provide a basis for breeding resistant varieties of $A$. andraeanum.

\section{Materials and Methods}

Bacterial strains and origin: $68 \mathrm{Xad}$ strains were isolated from infected A. andraeanum, which were collected from Guangzhou, Conghua, Shaoguan, Shunde, Xinfeng and Shanghai. Details of the Xad strains examined in this study are provided in Table 1.

Table 1. Xad strains, cultivars and origin.

\begin{tabular}{|c|c|c|c|c|c|}
\hline Xad Strains & Anthurium cultivars & Origin & Xad Strains & Anthurium cultivars & Origin \\
\hline $\mathrm{Xad1}, \mathrm{Xad} 2$ & A. feudleri & Conghua & Xad36, Xad37 & A. andraeanum 'Ricado' & Conghua \\
\hline Xad3 Xad6 & A. andraeanum 'Dakota' & Conghua & Хad38, Хad39 & A. andraeanum 'Kaxino' & Conghua \\
\hline $\mathrm{Xad7}, \mathrm{Xad} 8$ & A. itanhaense & Conghua & Xad40, Xad41 & A. andraeanum 'Luosa' & Conghua \\
\hline Xad9 Xad13 & A. andraeanum 'Sharade' & Conghua & Xad42 & A. andraeanum 'Windward' & Conghua \\
\hline Xad14, Xad15 & A. andraeanum 'Senator' & Conghua & Xad43, Xad44 & A. andraeanum 'Baron' & Conghua \\
\hline Xad16, Xad17 & A. andraeanum 'Madural' & Conghua & Xad45, Xad46 & A. andraeanum 'Sandy' & Conghua \\
\hline $\mathrm{Xad}$ & A. andraeanum 'Red Queen' & Conghua & 8 & $\begin{array}{l}\text { A. andraeanum } \\
\text { 'Pink Champion' }\end{array}$ & $\mathrm{Cc}$ \\
\hline Xad20 & A. andraeanum 'Sun fire' & Conghua & Xad49, Xad50 & A. andraeanum 'Fantasy Love' & Conghua \\
\hline Xad21, Xad22 & A. crassinervium & Conghua & Xad51, Xad52 & A. andraeanum 'Alabama' & Conghua \\
\hline $\mathrm{Xad} 23, \mathrm{Xad} 24$ & A. andraeanum 'Dovetail Red' & Conghua & Xad53 & A. andraeanum 'Mating' & Conghua \\
\hline $\mathrm{Xad} 25, \mathrm{Xad} 26$ & A. andraeanum 'Catherine' & Conghua & Xad54 Xad56 & A. andraeanum 'Vito' & Sunde \\
\hline Xad27 & A. andraeanum 'Pistache' & Conghua & Xad57 & A. andraeanum 'Alabama' & Sanshui \\
\hline Xad28, Xad29 & A040 & Conghua & Xad58, Xad59 & A. andraeanum 'Dakota' & Shaoguan \\
\hline Xad30, Xad31 & A. andraeanum 'Toscane & Conghua & Xad60 Xad62 & A. andraeanum 'Alabama' & Xinfeng \\
\hline Xad32, Xad33 & A. andraeanum 'Crystal Candle' & Conghua & Xad63, Xad64 & A. andraeanum 'Ardour' & Guangzhou \\
\hline Xad34, Xad35 & A. andraeanum 'Arebo' & Conghua & $\begin{array}{c}\text { Xad65 } \\
\text { Xad66 Xad68 }\end{array}$ & $\begin{array}{l}\text { A. andraeanum 'Alabama' } \\
\text { A. andraeanum 'Dakota' }\end{array}$ & $\begin{array}{l}\text { Guangzhou } \\
\text { Shanghai }\end{array}$ \\
\hline
\end{tabular}


Differential varieties of $A$. andraeanum: Five varieties of $A$. andraeanum, Vita, Red Victory, Pink Champion, Alabama and A. andraeanum 'Arebo' , all of which have shown different levels of resistance to Xad during the planting process for many years, were selected as differential hosts. The anthurium seedlings were planted in pots and were inoculated at the fourth-leaf stage.

Inoculation of $A$. andraeanum seedlings: Suspensions of Xad were inoculated onto anthurium seedlings by both leaf-cutting and foliar spray application. For leaf-cutting inoculation, the tips of two similarly-sized leaves from each plant were cut off using sterilized scissors and the bacterial suspensions were then evenly sprayed onto the wounded leaves. For spraying inoculation, the bacterial suspensions were sprayed directly onto unwounded leaves. Control plants were inoculated with sterile water. Each of the $68 \mathrm{Xad}$ strains was individually inoculated onto the leaves of the five different varieties of $A$. andraeanum, with each bacterial strain inoculated onto 10 seedlings of each $A$. andraeanum variety. All bacterial suspensions were adjusted to a concentration of $1 \times 10^{8}$ colony-forming units $/ \mathrm{mL}$. The inoculated anthurium seedlings were maintained in a greenhouse at $28^{\circ} \mathrm{C}$ and $>90 \%$ relative humidity.

Evaluation of disease severity: Disease progression was monitored weekly over the course of the experiment, with incidence and disease index values analyzed at 30 days post-inoculation.

The incidence of disease was equal to the number of diseased leaves divided by the number of total leaves $\times 100 \%$.

The grading standard of disease severity was as follows:

- Level 0: No diseased spots on the leaf.

- Level 1: The length of diseased spots was $2-3 \mathrm{~cm}$ or the area of the diseased spot was less than $10 \%$ of the leaf.

- Level 3: The length of diseased spots was less than $1 / 4$ of the leaf or the area of the diseased spot was less than $20 \%$ of the leaf.

- Level 5: The length of diseased spots was $1 / 4$ and less than $1 / 2$ of the leaf or the area of the diseased spot was between $20-49 \%$ of the leaf.

- Level 7: The length of diseased spots was $1 / 2$ and less than $3 / 4$ of the leaf or the area of the diseased spot was between $50-74 \%$ of the leaf.

- Level 9: The length of diseased spots was 3/4 or more of the leaf or the area of the diseased spot was over $75 \%$ of the leaf.

The disease index (DI), representing both the disease incidence and symptom severity, was calculated using the formula: $\mathrm{DI}=\left(\sum \mathrm{Di} \times \mathrm{Dd}\right) /(\mathrm{Mi} \times \mathrm{Md}) \times 100$, where $\mathrm{Di}$ is number of diseased leaves in a disease grade, $\mathrm{Dd}$ is the rating scale of the corresponding disease grade, $\mathrm{Mi}$ is the total number of leaves observed, and $\mathrm{Md}$ is the rating scale of the maximum disease grade.

Data integration and normalization: To more accurately classify the results of the experimental data, data normalization was conducted. Based on the experimental design and the obtained results, there were four groups of data: Disease incidence and disease index from both the foliar spray inoculation and the leaf-cutting inoculation. The data from each of the four groups were normalized and weighted for parametric analysis and an analytical hierarchy process was adopted.

According to the order of the original data, in most cases, the rank statistics of the incidence and disease indexes of the inoculated plants could be fitted using a discrete exponential analysis model as well as a logarithmic normalization method. Using A to represent the incidence coefficient, B for the disease index coefficient, $a$ for the incidence rate, and $b$ for the disease index, the following formulas were used:

$$
\mathrm{A}=\log _{100}(\mathrm{a} \times 100+1), \mathrm{B}=\log _{100}(\mathrm{~b}+1),(\mathrm{A} \in[0: 1] \mathrm{B} \in[0: 1]) .
$$

Hierarchical weightings: Using a simplified analytical hierarchy process, the weighting of each group of data at each level was determined. Usually, the determination of virulence and physiological race of plant pathogenic bacteria is based on wound inoculation, with the disease index being an important standard. Therefore, when integrating the results of wound and spray inoculation, we gave 
wound inoculation a higher weighting (70\%) than spray inoculation $(30 \%)$, with the disease index given a weighting of $70 \%$ and the incidence a weighting of $30 \%$ (Figure 1 ).

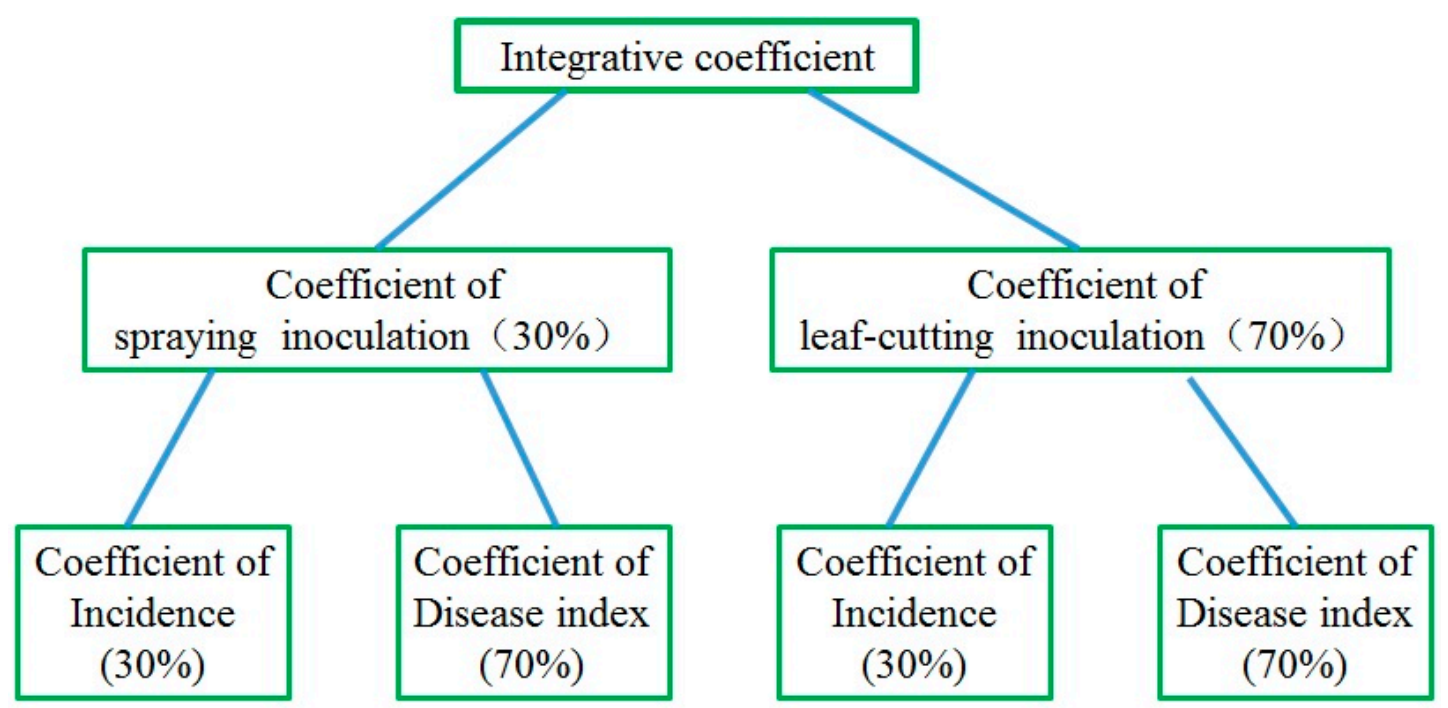

Figure 1. The hierarchy frame and weighting (\%) of the integrative coefficient. Coefficients at each level: Spraying/leaf-cutting inoculation coefficient $=0.3 \times$ incidence coefficient $+0.7 \times$ disease index coefficient. Integrated coefficient $=0.3 \times$ coefficient of spraying inoculation $+0.7 \times$ coefficient of leaf-cutting inoculation.

Data classification and resistance/susceptibility determination: The integrated coefficient values were calculated according to the above formula, which then allowed each pathotype to be classified as either resistant $(R)$ or susceptible (S). A pathotype was classified as resistant when the integrated coefficient value was between 0 and 0.5 , while susceptible pathotypes were those with an integrated coefficient between 0.5 and 1. In this way, the corresponding pathotype of each Xad strain was obtained via statistical analysis and subsequently classified.

\section{Results}

\subsection{Effects of Inoculation Method on the Virulence of Xanthomonas axonopodis pv. dieffenbachiae}

At 30 days post-inoculation, differences in disease incidence and disease index were noted among the different anthurium varieties and Xad strains. The incidence of disease in the five anthurium varieties following foliar spray inoculation ranged from $0-38.89 \%$, with disease index values ranging from $0-31.48$. In contrast, following leaf-cut inoculation, the disease incidence ranged from $6.25-100 \%$, with disease index values ranging from 0.69-98.52. The lowest average disease incidence and index values $(4.11 \%$ and 0.85 , respectively) were calculated for resistant the $A$. andraeanum variety Vita, while the highest average disease incidence and index values ( $78.2 \%$ and 57.42 , respectively) were determined for the susceptible variety, A. andraeanum 'Arebo' . However, in the leaf-cutting inoculation experiment, $\mathrm{Xad}$ strains showed differences in virulence against different anthurium varieties, while the same variety of $A$. andraeanum could also show different levels of resistance to different Xad strains. Overall, the disease incidence and index values were generally higher in the leaf-cutting assay compared with those obtained following foliar spray inoculation (Table 2). 
Table 2. Disease investigation after spraying and leaf-cutting inoculation after 30 days.

\begin{tabular}{|c|c|c|c|c|c|}
\hline \multirow[b]{2}{*}{ Xad Strains } & \multicolumn{5}{|c|}{ Varieties (Disease Index/Disease Incidence\%) } \\
\hline & $\begin{array}{c}\text { A. andraeanum } \\
\text { 'Vita' }\end{array}$ & $\begin{array}{l}\text { A. andraeanum } \\
\text { 'Red Victory' }\end{array}$ & $\begin{array}{l}\text { A. andraeanum } \\
\text { 'Pink Champion' }\end{array}$ & $\begin{array}{l}\text { A. andraeanum } \\
\text { 'Alabama' }\end{array}$ & $\begin{array}{l}\text { A. andraeanum } \\
\text { 'Arebo' }\end{array}$ \\
\hline \multirow{2}{*}{ Xad1 } & $0.00 / 0.00^{a}$ & $0.65 / 5.88$ & $0.74 / 6.67$ & $0.46 / 4.17$ & $14.81 / 14.81$ \\
\hline & $13.58 / 44.44^{\mathrm{b}}$ & $33.33 / 88.89$ & $67.28 / 100.00$ & $3.70 / 33.33$ & $34.64 / 100.00$ \\
\hline \multirow{2}{*}{$\mathrm{Xad} 2$} & $3.09 / 5.59$ & $0.00 / 0.00$ & $0.51 / 4.55$ & $15.79 / 15.79$ & $0.74 / 6.67$ \\
\hline & $24.31 / 43.75$ & $45.83 / 87.50$ & $47.22 / 87.50$ & $37.78 / 60.00$ & $26.39 / 75.00$ \\
\hline \multirow[b]{2}{*}{ Xad3 } & $0.00 / 0.00$ & $3.70 / 22.22$ & $4.89 / 12.00$ & $0.51 / 4.55$ & $2.02 / 9.09$ \\
\hline & $11.11 / 46.67$ & $25.93 / 86.67$ & $75.69 / 93.75$ & $28.57 / 42.86$ & $25.69 / 68.75$ \\
\hline \multirow{2}{*}{ Xad4 } & $1.39 / 6.25$ & $0.00 / 0.00$ & $0.53 / 4.76$ & $1.43 / 6.45$ & $1.35 / 12.12$ \\
\hline & $0.69 / 6.25$ & $8.15 / 46.67$ & $42.86 / 100.00$ & $14.81 / 53.33$ & $18.51 / 73.33$ \\
\hline \multirow[b]{2}{*}{ Xad5 } & $1.11 / 10.00$ & $6.88 / 33.33$ & $26.39 / 37.50$ & $31.48 / 38.89$ & $9.66 / 34.78$ \\
\hline & $31.11 / 53.33$ & $48.15 / 60.00$ & $59.26 / 80.00$ & $50.37 / 66.67$ & $68.89 / 86.67$ \\
\hline \multirow{2}{*}{ Xad6 } & $0.00 / 0.00$ & $0.00 / 0.00$ & $0.00 / 0.00$ & $0.53 / 4.76$ & $21.53 / 43.75$ \\
\hline & $20.74 / 26.67$ & $30.37 / 46.67$ & $46.67 / 60.00$ & $16.67 / 25.00$ & $76.39 / 100.00$ \\
\hline \multirow[b]{2}{*}{ Xad7 } & $1.15 / 3.44$ & $3.85 / 3.85$ & $0.40 / 3.57$ & $0.43 / 3.85$ & $3.23 / 16.13$ \\
\hline & $9.63 / 46.67$ & $20.00 / 73.33$ & $69.84 / 85.71$ & $57.78 / 80.00$ & $43.58 / 43.58$ \\
\hline \multirow{2}{*}{ Xad8 } & $0.00 / 0.00$ & $5.56 / 27.78$ & $9.44 / 15.00$ & $6.35 / 9.52$ & $4.70 / 42.31$ \\
\hline & $1.48 / 13.33$ & $57.04 / 100.00$ & $49.31 / 68.75$ & $25.40 / 42.86$ & $39.26 / 86.67$ \\
\hline \multirow{2}{*}{ Xad9 } & $0.00 / 0.00$ & $1.31 / 11.76$ & $0.00 / 0.00$ & $0.00 / 0.00$ & $1.35 / 12.12$ \\
\hline & $2.96 / 26.67$ & $6.67 / 46.67$ & $42.96 / 53.33$ & $25.69 / 56.25$ & $18.75 / 68.75$ \\
\hline \multirow{2}{*}{ Xad10 } & $3.24 / 12.50$ & $4.83 / 26.09$ & $0.00 / 0.00$ & $1.93 / 8.70$ & $9.47 / 25.93$ \\
\hline & $17.78 / 40.00$ & $39.32 / 92.31$ & $64.10 / 84.62$ & $56.41 / 92.31$ & $65.19 / 100.00$ \\
\hline \multirow[b]{2}{*}{ Xad11 } & $0.00 / 0.00$ & $0.00 / 0.00$ & $0.00 / 0.00$ & $0.00 / 0.00$ & $2.56 / 23.08$ \\
\hline & $5.93 / 40.00$ & $50.00 / 100.00$ & $20.00 / 33.33$ & $23.46 / 55.56$ & $4.27 / 38.46$ \\
\hline \multirow[b]{2}{*}{ Xad12 } & $1.65 / 7.41$ & $1.78 / 8.00$ & 2.8111 .11 & $0.41 / 3.70$ & $6.30 / 16.67$ \\
\hline & $5.56 / 50.00$ & $33.33 / 100.0$ & $76.19 / 100.0$ & $16.24 / 38.46$ & $30.37 / 86.70$ \\
\hline \multirow[b]{2}{*}{ Xad13 } & $0.00 / 0.00$ & $4.94 / 33.33$ & $4.00 / 12.00$ & $2.78 / 5.00$ & $6.76 / 17.39$ \\
\hline & $8.33 / 50.00$ & $35.56 / 66.67$ & $69.44 / 87.50$ & $22.96 / 73.33$ & $22.22 / 75.00$ \\
\hline \multirow{2}{*}{ Xad14 } & $1.15 / 3.45$ & $1.23 / 11.11$ & $14.29 / 14.29$ & $19.05 / 19 / 05$ & $12.12 / 12.12$ \\
\hline & $15.28 / 50.00$ & $54.17 / 100.00$ & $90.12 / 100.00$ & $54.81 / 93.33$ & $40.17 / 100.00$ \\
\hline & $0.00 / 0.00$ & $1.11 / 10.00$ & $2.22 / 20.00$ & $0.53 / 4.76$ & $2.22 / 20.00$ \\
\hline Xad15 & $2.61 / 22.22$ & $18.52 / 73.33$ & $63.89 / 100.00$ & $11.11 / 37.50$ & $24.79 / 81.25$ \\
\hline & $0.00 / 0.00$ & $1.39 / 12.50$ & $0.00 / 0.00$ & $0.37 / 3.33$ & $0.72 / 6.45$ \\
\hline Xad16 & $2.96 / 26.67$ & $22.96 / 53.33$ & $57.78 / 66.67$ & $35.56 / 66.67$ & $27.41 / 73.33$ \\
\hline & $1.71 / 7.69$ & $0.43 / 3.85$ & $0.00 / 0.00$ & $0.85 / 7.69$ & $4.37 / 25.00$ \\
\hline Xad17 & $8.33 / 37.50$ & $29.63 / 80.00$ & $86.51 / 92.86$ & $49.63 / 73.33$ & $48.89 / 66.67$ \\
\hline & $0.43 / 3.84$ & $0.00 / 0.00$ & $0.85 / 7.69$ & $0.00 / 0.00$ & $4.76 / 21.43$ \\
\hline Xad18 & $6.67 / 33.33$ & $15.56 / 60.00$ & $70.94 / 84.62$ & $23.70 / 66.67$ & $25.49 / 76.47$ \\
\hline & $0.62 / 5.56$ & $1.75 / 15.79$ & $0.00 / 0.00$ & $1.39 / 12.50$ & $15.46 / 43.48$ \\
\hline Xad19 & $17.78 / 53.33$ & $20.00 / 60.00$ & $33.33 / 73.33$ & $27.41 / 33.33$ & $42.86 / 85.71$ \\
\hline & $1.85 / 8.33$ & $0.82 / 7.41$ & $1.01 / 9.09$ & $13.19 / 18.75$ & $0.93 / 8.33$ \\
\hline $\mathrm{Xad} 20$ & $2.22 / 20.00$ & $9.52 / 57.14$ & $58.33 / 75.00$ & $37.91 / 58.82$ & $35.42 / 68.75$ \\
\hline & $1.59 / 4.76$ & $5.88 / 5.88$ & $0.00 / 0.00$ & $2.88 / 18.52$ & $1.23 / 11.11$ \\
\hline Xad21 & $1.48 / 13.33$ & $6.67 / 33.33$ & $51.11 / 60.00$ & $14.07 / 46.67$ & $8.89 / 40.00$ \\
\hline & $0.44 / 4.00$ & $0.97 / 8.70$ & $0.00 / 0.00$ & $0.89 / 8.00$ & $3.07 / 20.69$ \\
\hline 122 & $0.74 / 6.67$ & $12.59 / 73.33$ & $55.56 / 73.33$ & $16.67 / 50.00$ & $19.26 / 80.00$ \\
\hline & $0.00 / 0.00$ & $2.02 / 9.09$ & $0.62 / 5.56$ & $0.00 / 0.00$ & $1.67 / 15.00$ \\
\hline $\mathrm{Xad} 23$ & $3.47 / 18.75$ & $3.92 / 25.53$ & $61.48 / 73.33$ & $9.63 / 20.00$ & $28.47 / 93.75$ \\
\hline & $2.65 / 14.29$ & $3.97 / 14.29$ & $1.28 / 3.85$ & $12.35 / 22.22$ & $15.95 / 23.33$ \\
\hline Xad24 & $8.15 / 33.33$ & $11.11 / 46.57$ & $51.85 / 80.00$ & $14.29 / 28.57$ & $29.86 / 81.25$ \\
\hline & $0.97 / 8.70$ & $4.63 / 8.88$ & $0.00 / 0.00$ & $1.33 / 12.00$ & $0.00 / 0.00$ \\
\hline $\mathrm{Xad} 25$ & $16.67 / 38.89$ & $51.11 / 100.00$ & $65.81 / 84.62$ & $35.56 / 81.25$ & $31.94 / 87.50$ \\
\hline & $0.41 / 3.70$ & $0.43 / 3.85$ & $0.00 / 0.00$ & $4.98 / 17.24$ & $3.37 / 24.24$ \\
\hline $\mathrm{Xad} 26$ & $14.81 / 40.00$ & $26.50 / 100.00$ & $58.73 / 85.71$ & $25.40 / 78.57$ & $35.56 / 93.33$ \\
\hline & $0.00 / 0.00$ & $0.00 / 0.00$ & $5.22 / 11.76$ & $0.00 / 0.00$ & $1.75 / 15.79$ \\
\hline Xad27 & $7.64 / 35.00$ & $20.00 / 76.47$ & $82.22 / 100.00$ & $11.81 / 17.65$ & $43.06 / 64.29$ \\
\hline & $0.00 / 0.00$ & $0.00 / 0.00$ & $0.00 / 0.00$ & $0.00 / 0.00$ & $0.00 / 0.00$ \\
\hline $\mathrm{Xad} 28$ & $10.37 / 66.67$ & $38.52 / 78.57$ & $51.11 / 73.33$ & $51.28 / 92.31$ & $20.74 / 66.67$ \\
\hline & $0.00 / 0.00$ & $0.82 / 7.41$ & $4.55 / 4.55$ & $0.00 / 0.00$ & $0.85 / 7.69$ \\
\hline $\mathrm{Xad} 29$ & $1.48 / 13.33$ & $18.52 / 57.14$ & $45.24 / 60.00$ & $1.48 / 13.33$ & $30.37 / 73.33$ \\
\hline
\end{tabular}


Table 2. Cont.

\begin{tabular}{|c|c|c|c|c|c|}
\hline \multirow[b]{2}{*}{ Xad Strains } & \multicolumn{5}{|c|}{ Varieties (Disease Index/Disease Incidence\%) } \\
\hline & $\begin{array}{c}\text { A. andraeanum } \\
\text { 'Vita' }\end{array}$ & $\begin{array}{l}\text { A. andraeanum } \\
\text { 'Red Victory' }\end{array}$ & $\begin{array}{l}\text { A. andraeanum } \\
\text { 'Pink Champion' }\end{array}$ & $\begin{array}{l}\text { A. andraeanum } \\
\text { 'Alabama' }\end{array}$ & $\begin{array}{l}\text { A. andraeanum } \\
\text { 'Arebo' }\end{array}$ \\
\hline \multirow{2}{*}{ Xad30 } & $0.00 / 0.00$ & $1.31 / 11.76$ & $1.11 / 10.00$ & $28.57 / 11.76$ & $1.85 / 16.67$ \\
\hline & $3.89 / 25.00$ & $15.56 / 66.67$ & $75.00 / 100.00$ & $51.63 / 76.47$ & $34.72 / 100.00$ \\
\hline \multirow{2}{*}{ Xad31 } & $1.33 / 4.00$ & $1.33 / 12.00$ & $0.00 / 0.00$ & $0.41 / 3.70$ & $4.37 / 17.86$ \\
\hline & $8.89 / 40.00$ & $29.63 / 80.00$ & $90.64 / 100.00$ & $42.22 / 73.33$ & $37.50 / 87.50$ \\
\hline \multirow{2}{*}{ Xad32 } & $0.00 / 0.00$ & $0.00 / 0.00$ & $0.00 / 0.00$ & $0.48 / 4.35$ & $1.48 / 13.33$ \\
\hline & $13.89 / 37.50$ & $34.26 / 58.33$ & $29.17 / 50.00$ & $33.33 / 50.00$ & $16.30 / 53.33$ \\
\hline \multirow{2}{*}{ Xad33 } & $3.51 / 10.53$ & $0.51 / 4.55$ & $5.31 / 13.04$ & $1.85 / 16.67$ & $3.03 / 27.27$ \\
\hline & $3.47 / 18.75$ & $19.05 / 85.71$ & $65.87 / 86.67$ & $19.26 / 53.33$ & $30.37 / 100.00$ \\
\hline \multirow[b]{2}{*}{ Xad34 } & $0.00 / 0.00$ & $0.46 / 4.17$ & $5.80 / 17.49$ & $2.53 / 13.64$ & $0.00 / 0.00$ \\
\hline & $3.70 / 20.00$ & $42.22 / 73.33$ & $98.41 / 100.00$ & $26.39 / 62.50$ & $33.33 / 100.00$ \\
\hline \multirow{2}{*}{ Xad35 } & $0.53 / 4.76$ & $0.46 / 4.17$ & $2.65 / 14.29$ & $1.33 / 12.00$ & $3.29 / 22.22$ \\
\hline & $4.44 / 40.00$ & $28.89 / 86.67$ & $57.04 / 86.67$ & $26.67 / 80.00$ & $20.00 / 86.67$ \\
\hline \multirow[b]{2}{*}{ Xad36 } & $2.22 / 13.33$ & $1.01 / 9.09$ & $4.70 / 19.23$ & $0.00 / 0.00$ & $0.00 / 0.00$ \\
\hline & $5.19 / 33.33$ & $14.81 / 66.67$ & $53.33 / 80.00$ & $13.33 / 40.00$ & $21.48 / 73.33$ \\
\hline \multirow{2}{*}{ Xad37 } & $0.00 / 0.00$ & $0.00 / 0.00$ & $0.00 / 0.00$ & $10.00 / 10.00$ & $2.31 / 4.17$ \\
\hline & $3.92 / 35.29$ & $62.50 / 87.50$ & $94.44 / 100.00$ & $46.53 / 68.74$ & $37.04 / 100.00$ \\
\hline \multirow[b]{2}{*}{ Xad38 } & $3.33 / 30.00$ & $1.59 / 14.29$ & $0.00 / 0.00$ & $23.08 / 23.08$ & $0.00 / 0.00$ \\
\hline & $9.72 / 50.00$ & $12.59 / 56.67$ & $24.44 / 33.33$ & $35.56 / 48.00$ & $42.96 / 80.00$ \\
\hline \multirow{2}{*}{ Xad39 } & $0.41 / 3.70$ & $0.44 / 4.00$ & $1.23 / 3.70$ & $4.94 / 14.81$ & $3.81 / 22.86$ \\
\hline & $3.70 / 33.33$ & $21.48 / 73.33$ & $72.22 / 83.33$ & $36.51 / 85.71$ & $48.72 / 100.00$ \\
\hline \multirow[b]{2}{*}{$\mathrm{Xad} 40$} & $0.00 / 0.00$ & $0.53 / 4.76$ & $1.45 / 4.35$ & $12.82 / 23.08$ & $4.55 / 13.64$ \\
\hline & $5.19 / 33.33$ & $25.40 / 71.43$ & $70.37 / 83.33$ & $13.49 / 35.71$ & $37.50 / 87.50$ \\
\hline \multirow{2}{*}{ Xad41 } & $2.22 / 10.00$ & $2.42 / 13.04$ & $1.59 / 7.69$ & $0.85 / 7.69$ & $4.53 / 25.93$ \\
\hline & $6.94 / 37.50$ & $19.66 / 84.62$ & $51.85 / 66.67$ & $18.25 / 50.00$ & $35.04 / 84.62$ \\
\hline \multirow{2}{*}{$\mathrm{Xad} 42$} & $0.00 / 0.00$ & $0.00 / 0.00$ & $0.00 / 0.00$ & $0.46 / 4.17$ & $1.48 / 13.33$ \\
\hline & $3.70 / 20.00$ & $14.07 / 73.33$ & $43.65 / 64.29$ & $10.42 / 43.75$ & $14.07 / 60.00$ \\
\hline \multirow{2}{*}{$\mathrm{Xad} 43$} & $0.43 / 3.85$ & $0.00 / 0.00$ & $0.79 / 7.14$ & $0.00 / 0.00$ & $0.71 / 6.90$ \\
\hline & $7.41 / 40.00$ & $6.67 / 33.33$ & $74.81 / 100.00$ & $27.08 / 56.25$ & $25.93 / 73.33$ \\
\hline & $1.52 / 13.64$ & $2.22 / 10.00$ & $3.89 / 5.00$ & $0.00 / 0.00$ & $0.93 / 8.33$ \\
\hline Xad44 & $5.56 / 37.50$ & $12.50 / 62.50$ & $82.22 / 100.00$ & $31.85 / 73.33$ & $30.07 / 94.12$ \\
\hline & $0.37 / 3.33$ & $0.00 / 0.00$ & $1.11 / 10.00$ & $0.43 / 3.85$ & $0.74 / 6.67$ \\
\hline $\mathrm{Xad} 45$ & $5.19 / 33.33$ & $28.15 / 80.00$ & $60.00 / 86.67$ & $29.91 / 53.85$ & $22.22 / 80.00$ \\
\hline & $0.00 / 0.00$ & $0.00 / 0.00$ & $0.00 / 0.00$ & $2.56 / 7.69$ & $2.22 / 12.00$ \\
\hline Xad46 & $3.70 / 33.33$ & $8.89 / 53.33$ & $11.11 / 33.33$ & $11.11 / 28.57$ & $15.56 / 60.00$ \\
\hline & $0.69 / 6.25$ & $1.23 / 11.11$ & $0.48 / 4.35$ & $1.11 / 10.00$ & $0.48 / 4.35$ \\
\hline Xad47 & $7.41 / 44.44$ & $29.63 / 80.00$ & $81.70 / 100.00$ & $35.29 / 70.59$ & $37.04 / 100.00$ \\
\hline & $0.82 / 7.41$ & $0.48 / 4.35$ & $0.00 / 0.00$ & $1.28 / 11.54$ & $2.67 / 16.00$ \\
\hline Xad48 & $2.78 / 25.00$ & $24.79 / 100.00$ & $62.75 / 82.36$ & $69.84 / 100.00$ & $33.33 / 75.00$ \\
\hline & $1.45 / 4.35$ & $1.10 / 9.09$ & $0.00 / 0.00$ & $1.06 / 8.70$ & $3.54 / 31.82$ \\
\hline Хad49 & $5.23 / 25.53$ & $17.78 / 66.67$ & $60.00 / 73.33$ & $35.42 / 56.25$ & $29.63 / 80.00$ \\
\hline & $2.32 / 11.11$ & $0.00 / 0.00$ & $2.22 / 4.17$ & $0.40 / 3.57$ & $1.11 / 10.00$ \\
\hline Xad50 & $21.48 / 46.67$ & $25.40 / 85.71$ & $93.06 / 100.00$ & $21.48 / 46.67$ & $63.70 / 93.33$ \\
\hline & $0.00 / 0.00$ & $5.31 / 13.04$ & $0.00 / 0.00$ & $25.69 / 31.25$ & $8.99 / 23.81$ \\
\hline Xad51 & $55.56 / 100.00$ & $83.70 / 100.00$ & $88.19 / 93.75$ & $28.15 / 66.67$ & $67.44 / 100.00$ \\
\hline & $0.00 / 0.00$ & $0.00 / 0.00$ & $0.00 / 0.00$ & $0.53 / 4.76$ & $0.00 / 0.00$ \\
\hline Xad52 & $0.69 / 6.25$ & $30.56 / 75.00$ & $71.85 / 100.00$ & $40.52 / 70.59$ & $45.68 / 100.00$ \\
\hline & $0.00 / 0.00$ & $0.00 / 0.00$ & $1.67 / 5.00$ & $1.52 / 13.64$ & $2.34 / 15.79$ \\
\hline Xad53 & $2.22 / 20.00$ & $2.96 / 26.67$ & $38.46 / 69.23$ & $20.74 / 53.33$ & $9.63 / 33.33$ \\
\hline & $0.37 / 3.33$ & $0.00 / 0.00$ & $0.85 / 7.69$ & $0.00 / 0.00$ & $1.53 / 13.79$ \\
\hline Хad54 & $3.92 / 35.29$ & $5.56 / 35.71$ & $15.56 / 33.33$ & $31.25 / 56.25$ & $24.79 / 69.23$ \\
\hline & $0.00 / 0.00$ & $4.86 / 18.75$ & $4.83 / 8.70$ & $4.27 / 23.08$ & $4.89 / 28.00$ \\
\hline Xad55 & $9.03 / 56.25$ & $38.16 / 100.00$ & $82.22 / 100.00$ & $27.41 / 73.33$ & $24.44 / 86.67$ \\
\hline & $2.47 / 7.41$ & $0.00 / 0.00$ & $0.00 / 0.00$ & $3.54 / 4.55$ & $1.48 / 13.33$ \\
\hline Xad56 & $9.52 / 28.57$ & $34.81 / 100.00$ & $90.28 / 100.00$ & $22.96 / 46.67$ & $33.33 / 87.50$ \\
\hline & $0.82 / 7.41$ & $0.51 / 4.55$ & $0.00 / 0.00$ & $0.00 / 0.00$ & $2.53 / 22.73$ \\
\hline Xad57 & $1.31 / 11.76$ & $7.64 / 68.75$ & $40.12 / 61.11$ & $15.69 / 58.82$ & $13.58 / 66.67$ \\
\hline & $0.62 / 5.56$ & $0.62 / 5.56$ & $1.48 / 13.33$ & $0.00 / 0.00$ & $7.11 / 24.00$ \\
\hline Xad58 & $3.92 / 23.53$ & $9.63 / 60.00$ & $79.26 / 100.0$ & $39.87 / 76.47$ & $19.26 / 80.00$ \\
\hline
\end{tabular}


Table 2. Cont.

\begin{tabular}{cccccc}
\hline \multirow{4}{*}{ Xad Strains } & \multicolumn{5}{c}{ Varieties (Disease Index/Disease Incidence\%) } \\
\cline { 2 - 6 } & $\begin{array}{c}\text { A. andraeanum } \\
\text { 'Vita' }\end{array}$ & $\begin{array}{c}\text { A. andraeanum } \\
\text { 'Red Victory' }\end{array}$ & $\begin{array}{c}\text { A. andraeanum } \\
\text { 'Pink Champion' }\end{array}$ & $\begin{array}{c}\text { A. andraeanum } \\
\text { 'Alabama' }\end{array}$ & $\begin{array}{c}\text { A. andraeanum } \\
\text { 'Arebo' }\end{array}$ \\
\hline \multirow{2}{*}{ Xad59 } & $0.00 / 0.00$ & $0.48 / 4.34$ & $0.41 / 3.70$ & $1.17 / 10.53$ & $1.65 / 14.81$ \\
& $21.57 / 88.24$ & $15.56 / 73.33$ & $41.67 / 50.00$ & $51.39 / 75.00$ & $61.40 / 100.00$ \\
Xad60 & $0.00 / 0.00$ & $0.93 / 8.33$ & $1.45 / 13.04$ & $0.00 / 0.00$ & $3.57 / 32.14$ \\
& $4.86 / 43.75$ & $10.37 / 53.33$ & $45.93 / 66.67$ & $40.28 / 50.00$ & $11.11 / 64.71$ \\
Xad61 & $0.00 / 0.00$ & $0.00 / 0.00$ & $0.62 / 5.56$ & $0.51 / 4.55$ & $2.22 / 20.00$ \\
& $10.42 / 43.75$ & $11.85 / 53.33$ & $32.40 / 66.67$ & $23.61 / 50.00$ & $9.72 / 50.00$ \\
Xad62 & $9.52 / 9.52$ & $0.00 / 0.00$ & $0.00 / 0.00$ & $0.00 / 0.00$ & $1.15 / 10.34$ \\
& $23.61 / 75.0$ & $40.74 / 86.67$ & $45.83 / 75.00$ & $51.39 / 75.00$ & $39.58 / 81.25$ \\
Xad63 & $0.00 / 0.00$ & $0.00 / 0.00$ & $1.11 / 10.00$ & $0.00 / 0.00$ & $0.48 / 4.35$ \\
& $2.22 / 20.00$ & $26.39 / 75.00$ & $35.56 / 53.33$ & $4.44 / 26.67$ & $20.83 / 75.00$ \\
Xad64 & $0.93 / 8.33$ & $0.00 / 0.00$ & $0.00 / 0.00$ & $0.00 / 0.00$ & $0.82 / 7.41$ \\
& $2.97 / 26.67$ & $17.04 / 60.00$ & $67.41 / 86.67$ & $32.48 / 61.54$ & $24.31 / 68.75$ \\
Xad65 & $2.47 / 11.11$ & $4.94 / 22.22$ & $10.37 / 13.33$ & $1.75 / 15.79$ & $1.33 / 12.00$ \\
& $17.78 / 53.33$ & $57.14 / 85.71$ & $95.07 / 100.00$ & $28.89 / 60.00$ & $46.83 / 92.86$ \\
Xad66 & $0.00 / 0.00$ & $0.85 / 7.69$ & $8.00 / 8.00$ & $5.98 / 15.38$ & $2.42 / 21.74$ \\
& $14.07 / 60.00$ & $37.50 / 87.50$ & $98.52 / 100.00$ & $40.00 / 66.67$ & $65.93 / 100.00$ \\
Xad67 & $0.93 / 8.33$ & $0.51 / 4.55$ & $0.69 / 6.25$ & $1.11 / 10.00$ & $2.22 / 20.00$ \\
& $1.96 / 17.65$ & $8.15 / 60.00$ & $35.71 / 64.29$ & $38.56 / 76.47$ & $23.53 / 82.35$ \\
Xad68 & $0.43 / 3.85$ & $2.88 / 11.11$ & $0.47 / 4.17$ & $1.33 / 4.00$ & $10.32 / 39.29$ \\
& $15.97 / 56.25$ & $25.64 / 92.31$ & $64.29 / 92.86$ & $27.78 / 83.33$ & $55.56 / 87.50$ \\
\hline
\end{tabular}

${ }^{a}$ Disease index/disease incidence $\%$ by spraying inoculation. ${ }^{b}$ Disease index/disease incidence $\%$ by leafcutting inoculation.

\subsection{Determination of the Integrated Coefficient Values and Pathotypes of Xad Strains}

To allow for combined analysis of the leaf-cutting and foliar spray-based inoculation data, each of the data sets was given separate hierarchical weightings. The leaf-cutting inoculation data was given a rating of $70 \%$, while the spraying inoculation data was given a weighting of $30 \%$. In addition to considering the disease index, the incidence of disease was also considered in our analyses. As such, the disease index was assigned a weighting of $70 \%$, while the disease incidence was given a weighting of $30 \%$. Based on these weightings, the final integrated coefficient was calculated. As the normalized logarithmic values of the coefficients were used, the data showed a linear distribution with values between 0 and 1 (Table S1). In this study, the integrated coefficient values were used to classify pathotypes as either disease resistant $(R)$ or susceptible $(S)$, with values between 0 and 0.5 classified as resistant and those between 0.5 and 1 indicating a susceptible pathotype. After the determination of the resistant/susceptible phenotypes, the corresponding pathogenic type of each strain could be obtained through statistical analysis and computer-based classification. Based on these analyses, we determined that the $68 \mathrm{Xad}$ strains could be divided into seven pathotypes. The phenotypic characteristics of pathotype I, II, III, IV, V, VI and VII on the five anthurium differentiation varieties (Vita, Red Victory, Pink Champion, Alabama, A. andraeanum 'Arebo' ) were: RRRSS, RRSRS, RRSSS, RSRSR, RSSRS, RSSSS and SSSSS, respectively (Table 3 ). 
Table 3. The pathotypes of $68 \mathrm{Xad}$ strains.

\begin{tabular}{|c|c|c|c|c|c|c|c|}
\hline \multirow[b]{2}{*}{ Pathotypes } & \multicolumn{5}{|c|}{ Differentiation Varieties } & \multirow[b]{2}{*}{ Xad Strains } & \multirow[b]{2}{*}{ Total Strains } \\
\hline & $\begin{array}{l}\text { A. andraeanum } \\
\text { 'Vita' }\end{array}$ & $\begin{array}{l}\text { A. andraeanum } \\
\text { 'Red Victory' }\end{array}$ & $\begin{array}{l}\text { A. andraeanum } \\
\text { 'Pink Champion' }\end{array}$ & $\begin{array}{l}\text { A. andraeanum } \\
\text { 'Alabama' }\end{array}$ & $\begin{array}{l}\text { A. andraeanum } \\
\text { 'Arebo' }\end{array}$ & & \\
\hline I & $\mathrm{R}^{1}$ & $\mathrm{R}$ & $\mathrm{R}$ & $\mathrm{S}^{2}$ & S & Xad46 & 1 \\
\hline II & $\mathrm{R}$ & $\mathrm{R}$ & $\mathrm{S}$ & $\mathrm{R}$ & $\mathrm{S}$ & Xad23, Xad57, Xad42 & 3 \\
\hline III & $\mathrm{R}$ & $\mathrm{R}$ & S & $S$ & $\mathrm{~S}$ & $\begin{array}{c}\text { Xad4, Xad18, Xad43, Xad53, Xad54, Xad61, } \\
\text { Xad64, Xad67 }\end{array}$ & 8 \\
\hline IV & $\mathrm{R}$ & S & $\mathrm{R}$ & S & $\mathrm{R}$ & Xad11 & 1 \\
\hline $\mathrm{V}$ & $\mathrm{R}$ & $\mathrm{S}$ & $\mathrm{S}$ & $\mathrm{R}$ & $\mathrm{S}$ & Xad1, Xad15, Xad27, Xad29, Xad36, Xad63 & 6 \\
\hline VI & $\mathrm{R}$ & S & S & $S$ & $S$ & $\begin{array}{c}\text { Xad3, Xad6, Xad8, Xad9, Xad12, Xad13, } \\
\text { Xad16, Xad20, Xad21, Xad22, Xad28, Xad30, } \\
\text { Xad32, Xad33, Xad34, Xad35, Xad37, Xad39, } \\
\text { Xad40, Xad44, Xad45, Xad47, Xad48, Xad49, } \\
\text { Xad52, Xad55, Xad58, Xad60, Xad66 }\end{array}$ & 29 \\
\hline VII & $S$ & $S$ & S & $S$ & $S$ & $\begin{array}{c}\text { Xad2, Xad5, Xad7, Xad10, Xad14, Xad17, } \\
\text { Xad19, Xad24, Xad25, Xad26, Xad31, Xad38, } \\
\text { Xad41, Xad50, Xad51, Xad56, Xad59, Xad62, } \\
\text { Xad65, Xad68 }\end{array}$ & 20 \\
\hline
\end{tabular}




\subsection{Pathotype Analysis}

The results indicated that Xad strains belonging to pathotype VI or VII were the most prevalent and also the most virulent. Overall, $72 \%$ of the tested strains belonged to pathotype VI or VII. Of these, 29 strains isolated from 21 anthurium cultivars from five regions of China were classified as pathotype VI, while 20 strains from 15 anthurium cultivars from six regions were classified as pathotype VII. Pathotype I and II strains were less prevalent and showed the lowest levels of virulence in this study. These two pathotypes accounted for only $5.9 \%$ of all strains, amongst which, only Xad46 strain, isolated from the A. andraeanum cultivar Sandy in the Conghua region, was classified as pathotype I. Pathotype III, IV, and V strains were moderately virulent and accounted for $22.1 \%$ of the tested strains. Overall, eight, one, and six Xad strains were classified as pathotype III, IV, and V, respectively. Therefore, we hypothesize that the majority of Xad strains in China display a high level of virulence.

Interestingly, variation was observed in the virulence of Xad strains isolated from the same anthurium cultivar. For example, seven Xad strains isolated from the A. andraeanum cultivar Alabama were divided amongst four pathotypes (II, III, VI and VII), while nine strains isolated from Dakota cultivar plants were spread amongst pathotypes III, VI, and VII. In addition, we noted differences in the types and virulence levels of strains isolated from the same cultivar but from different geographical origins (Table 4). However, there was variation in strains from the same geographical origin. For example, in the Conghua area, $53 \mathrm{Xad}$ strains isolated from $26 \mathrm{~A}$. andraeanum cultivars were divided amongst the seven pathotypes (I VII) (Table 5 ) but pathotype VI and VII strains were predominant.

Table 4. Pathotypes of Xad strains from Alabama and Dakota in different geographical origin.

\begin{tabular}{cccc}
\hline Xad Strains & Anthurium cultivars & Origin & Pathotypes \\
\hline Xad57 & A. andraeanum 'Alabama' & Sanshui & II \\
Xad61 & A. andraeanum 'Alabama' & Xinfeng & III \\
Xad60 & A. andraeanum 'Alabama' & Xinfeng & VI \\
Xad52 & A. andraeanum 'Alabama' & Conghua & VI \\
Xad62 & A. andraeanum 'Alabama' & Xinfeng & VII \\
Xad65 & A. andraeanum 'Alabama' & Guangzhou & VII \\
Xad51 & A. andraeanum 'Alabama' & Conghua & VII \\
Xad4 & A. andraeanum 'Dakota' & Conghua & III \\
Xad67 & A. andraeanum 'Dakota' & Shanghai & III \\
Xad3, Xad6 & A. andraeanum 'Dakota' & Conghua & VI \\
Xad66 & A. andraeanum 'Dakota' & Shanghai & VI \\
Xad58 & A. andraeanum 'Dakota' & Shaoguan & VI \\
Xad5 & A. andraeanum 'Dakota' & Conghua & VII \\
Xad68 & A. andraeanum 'Dakota' & Shanghai & VII \\
Xad59 & A. andraeanum 'Dakota' & Shaoguan & VII \\
\hline
\end{tabular}


Table 5. Pathotypes of $53 \mathrm{Xad}$ strains from 26 cultivars in Conghua area.

\begin{tabular}{|c|c|c|}
\hline Xad Strains (Anthurium cultivars) & Pathotypes & Total Strains \\
\hline Xad46 (Sandy). & I & 1 \\
\hline Xad23 (Dovetail Red), Xad42 (Windward). & II & 2 \\
\hline Xad4 (Dakota), Xad18 (Red Queen), Xad43 (Baron), Xad53 (Mating). & III & 4 \\
\hline Xad11 (Sharada). & IV & 1 \\
\hline Xad1 (A. ieudleri), Xad15 (Senator), Xad27 (Pistache), Xad29 (A040), Xad36 (Ricado). & $\mathrm{V}$ & 5 \\
\hline $\begin{array}{l}\text { Xad3 (Dakota), Xad6 (Dakota), Xad8 (A. itanhaense), Xad9 (Sharada), Xad12 (Sharada), } \\
\text { Xad13 (Sharada), Xad16 (Madural), Xad20 (Sun fire), Xad21 (A. cassinervium), Xad22 } \\
\text { (A. cassinervium), Xad28 (A040), Xad30 (Toscane), Xad32 (Crystal Candle), Xad33 } \\
\text { (Crystal Candle), Xad34 (Arebo), Xad35 (Arebo), Xad37 (Ricado), Xad39 (Kaxino), } \\
\text { Xad40 (Luosa), Xad44 (Baron), Xad45 (Sandy), Xad47 (Pink Champion), Xad48 (Pink } \\
\text { Champion), Xad49 (Fantasy Love), Xad52 (A. andraeanum 'Alabama'). }\end{array}$ & VI & 25 \\
\hline $\begin{array}{l}\text { Xad2 (A. ieudleri), Xad7 (A. itanhaense), Xad10 (Sharada), Xad14 (Senator), Xad17 } \\
\text { (Madural), Xad19 (Red Queen), Xad25 (Catherine), Xad26 (Catherine), Xad31 } \\
\text { (Toscane), Xad38 (Kaxino), Xad41 (Luosa), Xad24 (Dovetail Red), Xad5 (Dakota), } \\
\text { Xad50 (Fantasy Love), Xad51 (A. andraeanum 'Alabama'). }\end{array}$ & VII & 15 \\
\hline
\end{tabular}

\section{Discussion}

Understanding the pathogenicity and differences in virulence of plant-pathogenic bacteria is a key factor in epidemic forecasting and in breeding disease-resistant plant varieties. Different anthurium cultivars display varying susceptibilities to the foliar and systemic phases of bacterial blight infection [15]. Resistance to both phases of infection is required to reduce the damaging effects of the pathogen. Understanding the genetic basis of both the resistance to blight in anthurium and the virulence of Xad is important for selecting parents for breeding and for designing an efficient anthurium breeding program [19]. A comprehensive analysis of 175 Xanthomonas axonopodis pv. dieffenbachiae strains isolated from 10 different Araceae hosts was previously conducted to assess pathogen variation [20]. The isolates were subjected to repetitive extragenic palindromic sequence polymerase chain reaction (Rep-PCR) analysis and four major phylogenetic clusters were generated. The results indicated that isolates grouped into cluster I, which were isolated primarily from anthurium cultivars, likely constituted an undescribed pathovar [20]. We later classified $66 \mathrm{Xad}$ strains isolated from 27 anthurium cultivars from Guangdong Province, China, into 14 genetic groups based on Rep-PCR analysis and identified obvious genetic differentiation amongst Xad strains in China [18]. We further investigated the virulence of $68 \mathrm{Xad}$ strains isolated from 30 different anthurium cultivars from China in the current study via foliar-spray and leaf-cutting inoculation of five different anthurium varieties. Our results indicated that the $68 \mathrm{Xad}$ strains belonged to seven different pathotypes, showing some correlation with genetic groups previously identified by Rep-PCR analysis [18]. Strains belonging to pathotype VI, isolated from 19 anthurium cultivars, and pathotype VII, isolated from 14 cultivars, were the most prevalent, accounting for $42.6 \%$ and $29.4 \%$ of all strains, respectively. Importantly, these two pathotypes were also associated with the highest levels of virulence in the current study.

We noted differences in the virulence of Xad strains isolated from the same cultivar but from different geographical locations (Table 4). Seven strains from the A. andraeanum cultivar Alabama belonged to four different pathotypes (II, III, VI, and VII), while nine strains from the cultivar Dakota were divided amongst pathotypes III, VI, and VII. The results also suggested that the majority of Xad strains isolated from cultivars Alabama and Dakota were highly virulent. While the 53 strains isolated from the Conghua area of Guangdong Province showed a high degree of pathotype diversity (pathotypes I-VII), strains belonging to pathotypes VI and VII were the most prevalent. Therefore, to prevent an outbreak of anthurium blight in the Conghua area, anthurium cultivar breeding should be aimed at developing resistance to pathotype VI and VII Xad strains. 
The inoculation method and selected disease severity scoring system are very important for assessing the virulence of pathogenic bacteria. Foliar infection occurs following pathogen entry into the leaves via hydathodes [21] or wounds [5]. In this study, two inoculation methods (leaf-cutting and spraying) were examined. The results showed that leaf-cutting was more effective than spraying for initiating disease, with some Xad strains showing virulence following leaf-cut inoculation but causing no symptoms in the foliar spray assays (Table 2). To allow for an integrated assessment of our results, we applied a weighting of $70 \%$ to the leaf-cut data versus $30 \%$ for the foliar spray data. Additionally, the disease index was given a weighting of $70 \%$, while the incidence of disease was assigned a weighting of $30 \%$. This allowed a final integrated assessment of coefficients and assignment of pathotypes into two categories: resistant and susceptible. Due to the integration of four sets of disease incidence and disease index data associated with two inoculation methods on five different varieties of $A$. andraeanum, we are confident that our results objectively and accurately reflect the virulence profiles of the $68 \mathrm{Xad}$ strains.

Supplementary Materials: The following are available online at http:/ /www.mdpi.com/2076-0817/7/4/85/s1, Table S1 showed the integrative coefficients and resistant/susceptible phenotypes of Xad strains on 5 anthurium varieties inoculation.

Author Contributions: Conceptualization, Q.-G.L. and X.-Y.Z.; Methodology, S.-C.L. and Q.-G.L.; Software, W.-D.Z.; Validation, Q.-G.L., S.-C.L. and X.-Y.Z.; Formal Analysis, S.-C.L.; Investigation, W.-D.Z. and X.-Y.Z.; Resources, W.-D.Z. and X.-Y.Z.; Data Curation, S.-C.L., and X.-W.L.; Writing-Original Draft Preparation, S.-C.L.; Writing-Review \& Editing, Q.-G.L.; Visualization, S.-C.L.; Supervision, Q.-G.L. and X.-Y.Z.; Project Administration, Q.-G.L. and X.-Y.Z.; Funding Acquisition, X.-Y.Z.

Funding: This research was supported by the Guangzhou fiscal agricultural project "Resistance identification and screening of anthurium varieties to Xanthomonas axonopodis pv. dieffenbachiae" (Guangzhou Agriculture (2013) no. 32).

Conflicts of Interest: The authors declare no conflicts of interest.

\section{References}

1. Hayward, A.C. A bacterial disease of anthurium in Hawaii. Plant Dis. Rep. 1972, 56, 904-908.

2. Nishijima, W.T. Anthurium blight: An overview. In Proceedings of the 1st Anthurium Blight Conference, Manoa, HI, USA, 23-25 February 1988; Alvarez, A.M., Ed.; pp. 6-8.

3. Elibox, W.; Umaharan, P. A green fluorescent protein-based screening method for identification of resistance in anthurium to systemic infection by Xanthomonas axonopodis pv. dieffenbachiae. Plant Pathol. 2007, 56, 819-827. [CrossRef]

4. Anonymous. EPPO standards-Diagnostic protocols for regulated pests PM7/23 Xanthomonas axonopodis pv. dieffenbachiae. Bull. OEPP 2004, 34, 183-186. [CrossRef]

5. Nishijima, W.T. Current anthurium blight control recommendations. In Proceedings of the 2nd Anthurium Blight Conference, Manoa, HI, USA, $22-24$ February 1989; Fernandez, J.A., Nishijima, W.T., Eds.; pp. 7-9.

6. Prior, P.; Hostachy, B.; Sunder, P.; Rott, P. Bacterial blight (X. campestris pv. dieffenbachiae) and bacterial leaf spot (Pseudomonas sp.) of anthurium in the French West Indies. Agron. Trop. 1985, 42, 61-68.

7. Ji, G.H.; Wei, Y.D.; Jiang, G.Z.; Guang, X.F.; Yu, S.F.; Liu, C.F. Preliminary identification of bacterial strain causing Anthurium bacterial blight. Acta Phytopathol. Sin. 2004, 34, 107-111.

8. Sathyanarayana, N.; Reddy, O.R.; Latha, S. Interception of Xanthomonas campestris pv. dieffenbachiae on Anthurium plants from the Netherlands. Plant Dis. 1998, 82, 262.

9. Isabelle, R.; Laurent, P.; Gagnevin, L. Specific detection of Xanthomonas axonopodis pv. dieffenbachiae in Anthurium (Anthurium andreanum) tissues by Nested PCR. Appl. Environ. Microbl. 2006, 72, 1072-1078.

10. Lu, M.L.; Zhou, X.Y.; Zeng, W.D.; Chen, Y.X.; Liu, Q.G. Specific PCR Detection of Xanthomonas axonopodis pv. dieffenbachiae. Acta Phytopathol. Sin. 2012, 42, 1-9.

11. Norman, D.J.; Alvarez, A.M. Monitoring the spread of Xanthomonas campestris pv. dieffenbachiae introduced from symptomless anthurium cuttings into production fields. J. Am. Soc. Hortic. Sci. 1996, 121, 582-585.

12. Anaïs, G.A.; Derrasse, P.P.; Cadic, A. Breeding anthuriums (Anthurium andraeanum L.) for resistance to bacterial blight caused by Xanthomonas campestris pv. dieffenbachiae. Acta Hortic. 2000, 508, 135-140. [CrossRef] 
13. Jiang, G.Z.; Zhao, L.F. Bactericides screening test on controlling bacterial leaf blight of Anthurium andraeanum. Trop. Agric. Sci. Technol. 2004, 27, 4-6.

14. Norman, D.J.; Henny, R.J.; Yuen, J.M.F. Resistance levels of pot anthurium cultivars to Xanthomonas campestris pv. dieffenbachiae. HortScience 1999, 34, 721-722.

15. Fukui, H.; Alvarez, A.M.; Fukui, R. Differential susceptibility of Anthurium cultivars to bacterial blight in foliar and systemic infection phases. Plant Dis. 1998, 82, 800-806. [CrossRef]

16. Seijo, T.E.; Peres, N.A.; Deng, Z.A. Characterization of Strains of Xanthomonas axonopodis pv. dieffenbachiae from Bacterial Blight of Caladium and Identification of Sources of Resistance for Breeding Improved Cultivars. HortScience 2010, 45, 220-224.

17. Elibox, W.; Umaharan, P. Inheritance of resistance to foliar infection by Xanthomonas axonopodis pv. dieffenbachiae in anthurium. Plant Dis. 2010, 94, 1243-1247. [CrossRef]

18. Fu, B.; Zhou, X.Y.; Luo, J.J.; Liu, Q.G. Analysis of the genetic diversity of Xanthomonas axonopodis pv. dieffenbachiae in Guangdong Province. J. Huazhong Agric. Univ. 2014, 33, 40-45.

19. Elibox, W.; Umaharan, P. Genetic basis of resistance to systemic infection by Xanthomonas axonopodis pv. dieffenbachiae in anthurium. Phytopathology 2008, 98, 421-426. [CrossRef] [PubMed]

20. Donahoo, R.S.; Jones, J.B.; Lacy, G.H.; Stromberg, V.K.; Norman, D.J. Genetic analyses of Xanthomonas axonopodis pv. dieffenbachiae strains reveal distinct phylogenetic groups. Phytopathology 2013, 103, 237-244. [PubMed]

21. Sakai, D.S. The effect of nitrate and ammonium fertilizer on the contents of anthurium guttation fluid. In Proceedings of the 3rd Anthurium Blight Conference, Manoa, HI, USA, 23-24 May 1990; Alvarez, A.M., Ed.; pp. 18-19.

(C) 2018 by the authors. Licensee MDPI, Basel, Switzerland. This article is an open access article distributed under the terms and conditions of the Creative Commons Attribution (CC BY) license (http:/ / creativecommons.org/licenses/by/4.0/). 